\title{
PROGRESS AND FUTURE DIRECTIONS IN BRIGHTNESS ELECTRON BEAM SOURCES*
}

\author{
X.J. Wang ${ }^{\dagger}$, ATF, NSLS, BNL, Upton, NY 11973, USA
}

\begin{abstract}
The progress of various high-brightness electron source technologies, specially laser based electron sources, such as photocathode RF gun, DC and pulsed photo-gun, and laser plasma electron source, will be reviewed. The physics processes of those sources to realize best performance are clarified according to three basic ideas. They are transverse space charge emittance compensation, space charge compensation and longitudinal emittance compensation. The major progresses and issues of photoinjector are briefly reviewed. The performance of photocathode RF gun at the Brookhaven Accelerator Test Facility (ATF) is presented.
\end{abstract}

\section{INTRODUCTION}

Since the discovery of the electron, electron beam (large number of electrons who are moving in the same direction with the same energy) has played a fundamental role in both pure scientific exploration and our daily life. The TV set in most homes, and the TV signal we watched are all driven by electron beam. Electron beam is one of the probes most widely used to study the structure of material(electron microscope), and explore the fundamental structure of the particles ( $\mathrm{e}^{-} \mathrm{e}^{+}, \mathrm{e}^{-\mathrm{p}}$ colliders). The quality of X-rays production improved dramatically only after the electron accelerators were introduced. The performance of electron beam for all those applications is determined by the quality of electron beam, and electron beam quality is largely determined by the electron sources.

Electrons can be produced in various ways, such as pair production from $\gamma$-ray. Electron sources in most applications are electron thermionic emission, field emission and photo-emission. Significant thermionic emission occurs only at relative high temperature (more than $10^{3}{ }^{\circ} \mathrm{C}$ ), the thermionic current density is low, and the time structure of the beam is long [1]. The presence of high field $(\mathrm{GeV} / \mathrm{m})$ leads to electrons tunnel through potential barrier, this is called field emission. Most field emission source relies on the local enhancement of the field using sharp tip. It is one the most active $R \& D$ area for vacuum electronics applications [2]. The low current of the field emission sources is the major limitation. The electron source we are going to discuss in this report will be produced by photo-emission process driven by the laser, we will also discuss other electron sources produced by laser interaction with either plasma or solid metal.

\footnotetext{
* Supported by US DOE contract DE-AC02-98CH10886 †xwang@bnl.gov
}

One of the most important laser based electron sources is photocathode RF gun [3]. It combines two welldeveloped technologies - high power laser and RF linac. Laser is used to produce and control the electron beam, and the high field in the RF cavity accelerates electron quickly to minimize the space charge effect. The advantage of photocathode RF gun is best demonstrated by the fact that, all saturations observed for the singlepass high-gain Free Electron Laser (FEL) are driven by a photocathode RF gun $[4,5,6]$.

In the following sections, we will first review three basic processes, transverse space-charge emittance compensation, space charge compensation, and longitudinal emittance compensation, which are used to produce brightness electron beam and experiments associated with them. Then we will discuss the major progress in photocathode RF gun technologies, specially laser and cathode performance. Finally we will outline several areas of brightness electron beam R\&D needs to be addressed, such as polarized $\mathrm{RF}$ gun and $\mathrm{CW}$ photocathode RF gun for energy recovery linac application[7].

\section{EMITTANCE COMPENSATION}

The quality of the electron beam is characterized by its brightness $B$ :

$$
B=\frac{N e}{2 \pi \ell \varepsilon_{n, x} \varepsilon_{n, y}}
$$

Where $\mathrm{N}$ is the number of the electrons; $\ell$ is the rms electron bunch length, $\varepsilon_{\mathrm{n}, \mathrm{x}}$ and $\varepsilon_{\mathrm{n}, \mathrm{y}}$ are normalized rms emittance of the electron beam. The simplified transverse envelope equation of the electron bunch can be written as [8],

$$
r^{\prime \prime}+k(s) r-\frac{2 r_{e} N / \ell}{r^{2}(\beta \gamma)^{3}} r-\frac{\varepsilon^{2}{ }_{n}}{(\beta \gamma)^{2} r^{3}}=0
$$

where $k(s)$ is the focusing of the transport line, $r_{e}$ is classical electron radius, $r$ is the rms beam size, and $\gamma$ is the electron beam relativistic energy. A different equation can also be written for the longitudinal dimension of the electron beam [9],

$$
\begin{aligned}
z^{\prime} & =\frac{\delta}{\gamma^{2}} \\
\delta^{\prime} & =\frac{3 r_{0} N}{\gamma^{3} \beta^{2} \ell_{b}^{3}} f(a, b) z
\end{aligned}
$$


where $\delta$ is the relative energy spread of the electron beam, $z$ is the rms bunch length and $f(a, b)$ is the electron beam form factor. Comparing equations (1), (2), and (3), we concluded that,

- The bright electron beam requires more charge, shorter bunch, and small transverse emittance, which are inherently incompatible with space charge effect.

- The most effective measure to reduce space charge effect is to increase the beam energy $\gamma$.

- The impact of the space charge effect on transverse and longitudinal distribution of the electron beam is quite different. A proper design of the transverse focusing channel can be used to control the transverse space charge effect. There is no effective longitudinal focusing to balance the space charge effect; ballistic compression and RF acceleration may be used for longitudinal space charge effect control with some limitations.

In the rest of this section, we will discuss three basic ideas have been used to minimized the emittance.

\subsection{Transverse space charge emittance compensation}

After introduction of photocathode RF gun, one of the most important techniques used to minimized the transverse emittance is so called transverse space charge emittance compensation [10]. The theoretical models were introduced later to formulate the transverse space charge emittance compensation [11]. The physics of the transverse space charge emittance compensation involves realignment of the transverse phase space along the electron beam longitudinal distribution caused by the space-charge defocusing effect longitudinal variation. This could be accomplished using a solenoid magnet, and it was directly experimental demonstrated at the BNL ATF (Fig.1) [12].

One of the consequences of the optimization of the transverse emittance is the increase of the longitudinal emittance of the electron beam because non-existence of the focusing in the longitudinal dimension (Fig.2). Fig. 2 plotted both transverse and longitudinal emittances for a $150 \mathrm{pC}$ charge as function of the distance for three settings of the emittance compensation solenoid magnet in a L-band photoinjector. The optimized transverse emittance for the solenoid magnet corresponds to the maximum longitudinal emittance.

The name of transverse space charge emittance compensation is very misleading in two aspects. First of the all, the emittance minimization is not truce space charge compensation (which has been introduced in ion beam community). Its behavior more like transverse phase space echo effect.
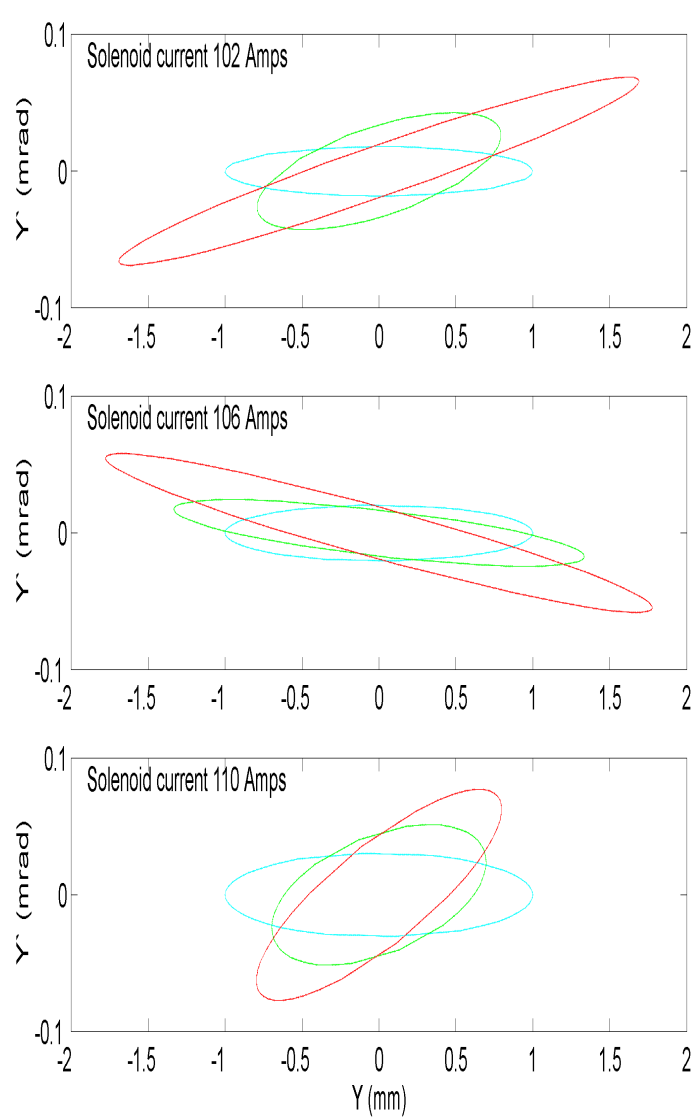

Figure1: Transverse emittance compensation using slice emittance measurements.

\subsection{Space charge emittance compensation}

The true space charge compensation should have the presence of the positive ions to neutralize the space charge effect. This effect was observed for both photocathode RF gun [13] and laser plasmas accelerators $[14,15,16,17]$.

The space charge compensation in photocathode RF gun (plasmas cathode) was first observed at the BNL ATF when laser was focused into a small spot, and the laser power density reached $\mathrm{GW} / \mathrm{cm}^{2}$ on cathode. The pulse length of the electron beam is determined by the RF frequency and field strength. Because of the small laser spot, and immovability of the ion, the transverse emittance are dominated by the RF effect. To produce electron beam with a bunch length less than 10 ps (FWHM) and peak current on the order $100 \mathrm{~A}$, higher frequency (X-band) photocathode RF gun operating at higher field (more than $150 \mathrm{MV} / \mathrm{m}$ ) is required.

Since LILAC was first introduced by University of Michigan group[14], several variations of the laser plasmas electron source were developed [15,16,17]. All those ideas takeing advantage of the high acceleration gradient and space charge compensation presence in the plasma. The issue for such injector is the short interaction distance of the acceleration field, and hence relative low energy. Relative low energy makes transverse emittance preservation difficult, and rapid electron beam bunch 
lengthening. Though electrons from plasmas injector were observed in several labs, there little information available in characterizing the electron beam quality. Emittance deduced from a single profile measurement may not correct due to the focusing (pinch) effect as electrons acquire energy.
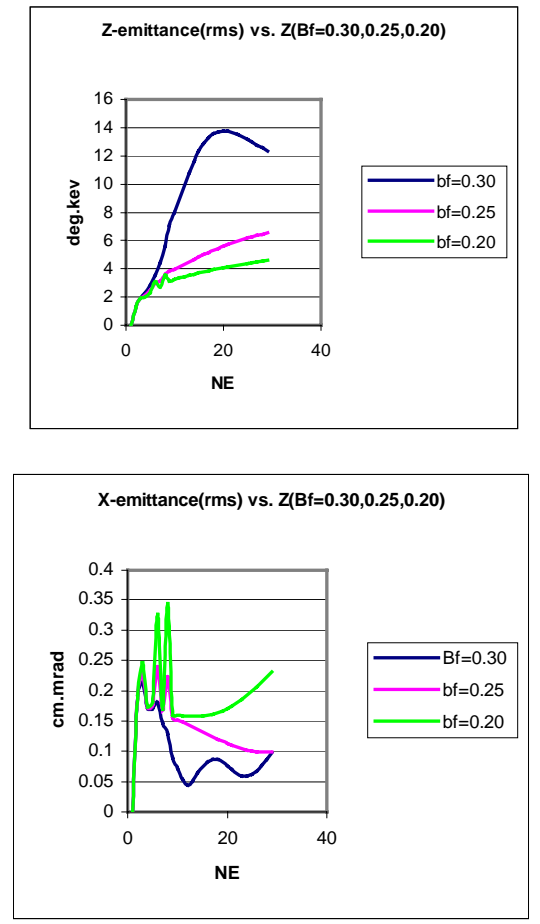

Figure 2: Longitudinal (top) and transverse (bottom) emittances as function of the distance and magnetic fields.

\subsection{Longitudinal Emittance emittance compensation}

One of the most common mis-understandings about photocathode RF gun is that, electron beam bunch length is determined by the laser pulse length and charge. Both simulation and experiment have shown that $[18,19]$, electron beam bunch length is also the function of the RF gun phase. The reduction of the electron beam bunch length leads to the reduction of overall transverse emittance is called longitudinal emittance compensation [20].

When laser incident on the photocathode, photoelectrons were produced promptly. Since electrons escape from the cathode surface with relative low energy, the photo-electron beam shaped like disk with large longitudinal compression comparing to laser pulse length initially. The initial electron beam expansion due to velocity difference will also be modified by the rate of the energy gain. The net effect will be the electron beam bunch length at the photocathode RF gun exit will depend on the RF gun phase and field strength. The energy and energy spread of the electron beam depend on the RF gun phase and field gradient on the cathode (Fig.3). Fig.3 shows the energy distribution as a function of the RF gun phase for three different field gradient, the change of the slope at the lower RF gun phase as field increase will lead to further electron beam bunch compression in the drift space following the photocathode RF gun for higher field.

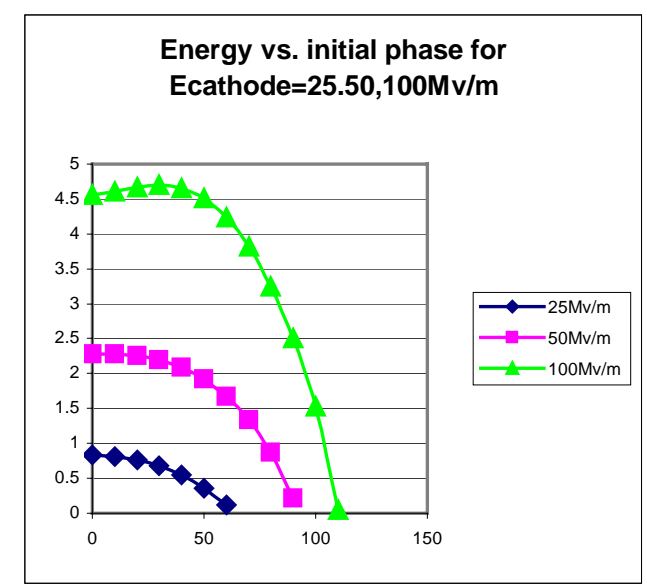

Figure 3: photo-electron beam energy as function of the RF gun phase and field gradient for BNL Gun IV.
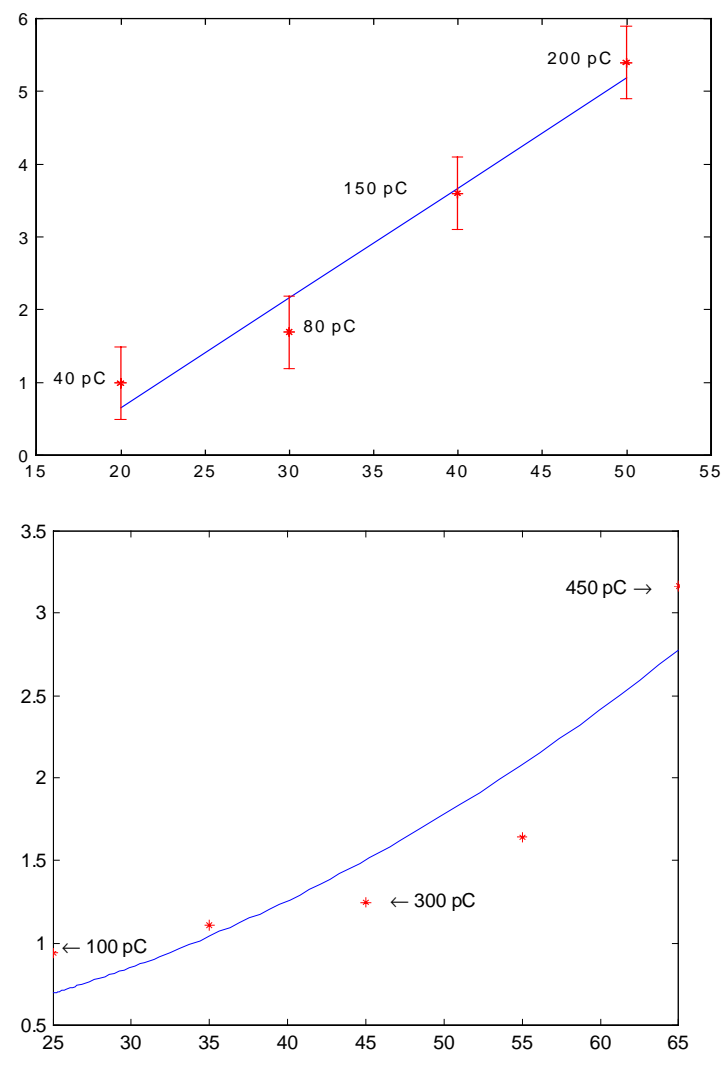

Figure 4: Photo-electron beam bunch length (top, FWHM (ps)) and normalized rms emittance (mm-mrad) as function of the RF gun phase. Crest of the field corresponding to 90-degree.

Fig.4 is the experiment measurements of the photoelectron beam bunch length and normalized rms emittance as functions of the RF gun phase. The measurements were performed with fixed laser energy, and the charge variation with RF gun phase are caused by the Schottky effect of the photo-emission. 
Operating at the lower RF gun phase for longitudinal emittance compensation is contrary to earlier RF gun theory [21] and simulation for transverse space charge emittance compensation [22]. Our recent simulation [23] now shown quality agreement with the prediction of the longitudinal emittance compensation. The Brookhaven Accelerator Test Facility (ATF) has been operating in this mode for the last six years, we now routinely have electron beam with normalized rms emittance less than 2 mm-mrad for a peak current of $100 \mathrm{~A}$. The best emittance measured for a $0.5 \mathrm{nC}$ charge is less $1 \mathrm{~mm}-\mathrm{mrad}$ [24].

\section{PROGRESS OF PHOTOINJECTOR TECHNOLOGIES}

Significant progress was made in many areas of photoinjector. We will briefly outline some major achievements in the photo-cathode brightness electron sources.

\subsection{Stability and Reliability}

One of the inherent character of the laser based electron sources is the stability and reliability of the electron beam because of the timing, spatial and intensity variation of the photocathode laser system. There are still significant gap between the electron beam stability and reliability we used to expect from thermionic electron gun, and the present laser system delivers.

The BNL ATF is the only multi-user facility in the world based on the photo-injector. It provided about 1100 hours user beam time annually. Our experience shown that, a stable and reliable photo-injector is the key to improve the quality of electron beam and productive facility. The stability and reliability of the photo-injector based facility are most determined by the laser and the operating environments. For a S-band photo-injector, delivered laser energy stability better than $10 \%$ (peak to peak), timing jitter less than $1 \mathrm{ps}$ (p-p) and point stability better than $1 \%$ are required.

\subsection{Photo-Injector System}

The introduction of the concept of photo-injector system will made photo-injector [25] much more productive. A photo-injector system should consists of photocathode RF gun, photocathode, emittance compensation solenoid magnet, laser and light transport system, electron beam and laser diagnostics. The photo-injector system should be capable of producing and measuring the basic laser and electron beam parameters, such as the three most basic parameters of operating a photocathode injector, photoelectron beam charge, electron beam energy and the RF gun phase laser arrives on the cathode.

\subsection{Photocathode}

One of the most important elements which determine the stability and reliability of the photo-injector is the photocathode. Robust high efficient photo-cathode will not only reduce the cost of the laser system, and also improve the stability and reliability of the laser system by reducing the heat load of the laser system. Significant progress was made in both semi-conductor and $\mathrm{Mg}$ cathodes.

Research carried out at CERN, INFN-Milano, LANL and many other groups have significantly improved the life time of semi-conductor photo-cathode, specially $\mathrm{Cs}_{2} \mathrm{Te}$ [26]. The life-time of the $\mathrm{Cs}_{2}$ Te cathode could now reach months with quantum efficiency on the order of a couple of percents. Experiments shown that, semiconductor cathode could be rejuvenated either by heating or ion bombardments.

We have developed a systematic procedure at the BNL ATF to manufacture; preparation and vacuum based cathode-cleaning technique for $\mathrm{Mg}$ cathode [27,28]. Using friction welding and proper surface contact, we now can reliably manufacture $\mathrm{Mg}$ cathode on the copper back plane. To remove the $\mathrm{Mg}$ cathode surface oxidation and contamination, we developed laser cathode cleaning technique without damage the cathode. We monitored the $\mathrm{RF}$ gun vacuum as the laser energy was adjusted. By keeping the RF gun cavity vacuum better than $10^{-9}$ Torr, we can achieve $100 \%$ laser cathode cleaning reliability. The $\mathrm{Mg}$ cathode now has operation QE $0.2 \%$ and lifetime longer than two years.

\subsection{Laser Pulse shaping}

One of the advantages of the laser driven brightness electron source is that, space charge effect could be controlled and minimized by proper laser pulse shaping. Both transverse and longitudinal uniformed laser pulse could reduce the emittance growth by space charge effect. Transverse laser profile could be controlled in several ways. From brute force truncation to more elaborate adaptive mirror. The structures of the laser profile introduced during the UV conversion is the major challenge.

Experiments are on-going in many labs to study the laser pulse longitudinal shaping using pulse stacking (SLAC GTF), frequency domain modulation (SHI) and saturable absorber (BNL ATF). The issue is what is the proper pulse shape for realizing the minimum transverse emittance. The uniformed laser pulse may not lead to the uniformed electron beam longitudinally due to space charge and the Schottky effects.

\subsection{Flat Beam}

Recent experiment demonstration of the flat beam production at the Fermilab A0 could open new applications for photo-injector [29,30]. The trick of the flat beam production consists of two parts. First make finite field present on the photo-cathode. And then the adaptive optics made up of skew quadrupole magnets transform round beam to a flat beam with large emittance ratio. The whole process preserves the product of the horizontal and vertical emittance. 


\section{FUTURE R\&D AND APPLICATIONS}

Predicting future is always a risk business, someone has to do it. Here is a brief list of items need to be addressed for future photo-injector $R \& D$ :

- Stability and reliability: without stability and reliability, photo-injector will never be able to realize its potential.

- What is the final limit of the photo-injector? Thermal, RF and space charge induced emittance, how they relate to each other?

- Laser and electron beam diagnostics: developing on-line laser and electron beam diagnostics will be rewarded for better performance of the photo-injector.

- Systematically comparing the predictions of computer code with experimental results

- All solid-state diode-pumped laser system and programmable laser pulse shaping system for photo-injector.

- Robust photocathode for visible and IR laser.

- Polarized photocathode RF gun.

- CW photocathode RF gun for energy recovery linac and other applications.

- Beam optimization for both transverse and longitudinal emittance.

Photocathode RF gun injector has demonstrated its superior performance and flexibility. Photo-electron beams with charge from $100 \mathrm{nC}$ to $\mathrm{pC}$, bunch length from 100 s femto-second to pico-seconds have been produced. One of the most important potential applications of the photo-injector is to retrofit existing synchrotron light source injectors. There are more than 70 light sources around the world; the injectors of those light sources are idle most time. Retrofit those injectors with photo-injector will yield several benefits with minimum investment. Better beam quality will reduce the radiation hazard. Photoelectron beam can be used to produce femtoseconds coherent IR radiation, or femto-seconds X-ray by laser Compton scattering. Photoelectron beam can also be used to drive FEL.

\section{ACKNOWLEDGEMENT}

Author would like to acknowledge many helpful discussions with colleague and collaborators from US, Japan, Europe and other countries. The most work discussed in this report performed at the Brookhaven Accelerator Test Facility, collaborations with all ATF coworkers, particularly with M. Babzien, I. Ben-Zvi, R. Malone and V. Yakimenko are gratefully acknowledged.

\section{REFERENCES}

[1] W.B. Herrmannsfeldt, "Low Emittance Thermionic Electron Gun", AIP Conference Proceeding No. 184, Edited by M. Month and M. Dienes, 1532 (1993).

[2] I Brodie and C.A. Spindt, "Vacuum Microelectronics" Advances in Electronics and Electro Physics 83, 2-106 (1992).

[3] J.S. Fraser, R.L. Sheffield and E.R. Gray, Nucl. Instrum. Methods Phys. Res. A, 250, 71(1986).

[4] L.H. Yu et al, Science, 289 (2000) 932-936.

[5] S. Milton et al, Science, 292 (2001) 2037-2041.

[6] A. Tremaine et al, Saturation Measurements of VISA, to be published.

[7] I. Ben-Zvi et al, NSLS PERL Project, this proceeding.

[8] M. Reiser, Theory and Design of Charged Particle Beams, Wiley, New York, 1994.

[9] A. Chao, Physics of Collective Beam Instabilities in High Energy Accelerators, Wiley, New York, 1993.

[10] B.E. Carlsten, Nucl. Instrum. Methods Phys. Res. A, 285, 313(1989).

[11]L. Serafini and J.B. Rosenzweig, Phys. Rev. E 55, 7565 (1997).

[12] X. Qiu, K. Batchelor, I. Ben-Zvi and X.J. Wang, Phys. Rev. Lett. 76, 3723-3726 (1996).

[13] X.J. Wang et al, J. Appl. Phys. 72, 888 (1992).

[14] D. Umstadter et al, Phys. Rev. Lett. 76, 2073 (1996).

[15] E. Esarey et al, Phys. Rev. Lett. 79, 2682 (1997).

[16] C. I. Moore et al, Phys. Rev. Lett. 82, 1688 (1999).

[17] Nasr Hafz et al, Nucl. Instrum. Methods Phys. Res. A, 455, 148 (2000).

[18] X.J. Wang, UCLA PhD thesis (1992).

[19] X.J Wang, X. Qiu and I. Ben-Zvi, Phys. Rev. E,54, R3121 - R3124 (1996).

[20] X.J. Wang and I. Ben-Zvi, Pro. Of PAC'97, 27932795(1997).

[21] K.J. Kim, Nucl. Instrum. Methods Phys. Res. A, 275, 201(1989).

[22] Juan Gallardo and Harold Kirk, BNL-61313 CAP 111-ATF-94C (1994).

[23]X.Y. Chang et al, this proceeding.

[24] V. Yakimenko, M. Babzien, I. Ben-Zvi and X.J. Wang, to be presented at FEL 2001.

[25]X.J. Wang, "Single-Pass High-Gain FEL Beam Diagnostics", Proceeding Of Beam Instrumentation Workshop 2000, AIP Conf. Proc. 546, 164-185 (2000).

[26] I. Boscolo and P. Michelato, Nucl. Instrum. Methods Phys. Res. A, 445, 389 (2000).

[27] X.J. Wang et al, Nucl. Instrum. Methods Phys. Res. A, 356, 159(1995).

[28] X.J. Wang et al, to be presented at FEL2001.

[29] R. Brinkmann et al, Phys. Rev. Special Topics accelerators and beam, 4, 053501 (2001).

[30] D. Edwards et al, this proceeding. 